López-Gajardo, M.A.; González-Ponce, I.; Pulido, J.J.; García-Calvo, T.; Leo, F.M. (2020) Analysis of the Technical-Tactical Actions by Goalkeeper on Football in Competition. Revista Internacional de Medicina y Ciencias de la Actividad Física y el Deporte vol. 20 (80) pp. 577-594 Http://cdeporte.rediris.es/revista/revista80/artanalisis1194.htm

DOI: https://doi.org/10.15366/rimcafd2020.80.008

\title{
ORIGINAL
}

\section{ANÁLISIS DE LAS ACCIONES TÉCNICO-TÁCTICAS DEL PORTERO DE FÚTBOL EN COMPETICIÓN}

\section{ANALYSIS OF THE TECHNICAL-TACTICAL ACTIONS BY GOALKEEPER ON FOOTBALL IN COMPETITION}

\author{
López-Gajardo, M.A. ${ }^{\text {; }}$ González-Ponce, I. ${ }^{2}$; Pulido, J.J. ${ }^{3}$; García-Calvo, T.4 \\ y Leo, F.M. ${ }^{5}$ \\ ${ }^{1}$ Contratado Pre-doctoral FPU, Facultad de Ciencias del Deporte, Universidad de Extremadura, \\ (España) malopezgajardo@unex.es \\ 2 Profesor Contratado Doctor, Facultad de Educación, Universidad de Extremadura (España) \\ ingopo@unex.es \\ 3 Contratado Post-doctoral, Facultad de Ciencias del Deporte, Universidad de Extremadura \\ (España) jjpulido@unex.es \\ ${ }^{4}$ Profesor Titular, Facultad de Ciencias del Deporte, Universidad de Extremadura (España) \\ tgarcíacalvo@unex.es \\ 5 Profesor Contratado Doctor, Facultad de Formación del Profesorado, Universidad de \\ Extremadura (España) franmilema@unex.es
}

Apoyo financiero proporcionado por el Fondo Europeo de Desarrollo Regional y, también por FSE, Junta de Extremadura (Consejería de Economía e Infraestructuras) [números de subvención GR18102, TA18027 y PO17012] y Gobierno de España (Ministerio de Educación, Cultura y Deporte) [números de subvención FPU17 / 03489]

Código UNESCO / UNESCO code: 6199 Psicología del deporte/ Sport Psychology

Clasificación Consejo de Europa / Council of Europe classification: 15. Psicología del deporte / Sport Psychology

Recibido 19 de noviembre de 2018 Received November 19, 2018 Aceptado 25 de abril de 2019 Accepted April 25, 2019

\section{RESUMEN}

El objetivo del presente estudio fue analizar las diferencias en el número y tipo de acciones técnico-tácticas de los porteros en competición en función de la división y de si juegan como local o visitante. Para ello, se han analizado 80 partidos con un total de 160 porteros de $1^{\underline{a}}, 2^{a}, 2^{a}-B$ y $3^{\underline{a}}$ división española. Los resultados mostraron que no existen diferencias significativas entre el número de acciones medias realizadas por partido en cada división, pero sí se encontraron 
diferencias significativas en 9 de los 48 gestos técnico-tácticos específicos estudiados. Además, se encontraron pequeñas diferencias en cuanto al número de acciones en función de si los porteros juegan como local o visitante. Por tanto, la principal conclusión de este estudio es la importancia de analizar los tipos de acciones técnico-tácticas de los porteros ya que nos pueden ofrecer información relevante para el entrenamiento y la competición.

PALABRAS CLAVE: Despeje, metodología observacional, saque de puerta.

\section{ABSTRACT}

The present study tries to respond about three different objectives: a) to quantify the number of technical-tactical actions carried out by goalkeepers during matches and to analyze if there are differences between divisions, $b$ ) to examine the possible differences in the number and type of technical-tactical actions between divisions under investigation, and c) to analyze the differences between this number and the type of technical-tactical actions when goalkeepers play at home team or away teams. To this end, a viewing was made of the 80 matches of the four most important divisions of Spanish football and a total of 160 goalkeepers were analyzed using the observational methodology. After the application of the tests the $\mathrm{H}$-test of Krustal-Wallis and $\mathrm{U}$ of Mann-Whitney, showed no significant differences between the number of average actions performed per game in each division. Only significant differences were found in 9 of the 48 specific technical-tactical gestures studied. So, the first conclusion in this study is the importance of analyzing the types of technical-tactical actions of goalkeepers, due to they can offer relevant information for training and competition.

KEY WORDS: Clearance, observational methodology, goal kick.

\section{INTRODUCCIÓN}

En las últimas décadas, el fútbol se ha convertido en objeto de estudio para los científicos del deporte. Al ser un deporte colectivo, comprende un estudio más complejo que los deportes individuales, porque los factores que afectan al rendimiento son mayores y más amplios (Palau, López y López, 2010). Por tanto, hay que tener en cuenta los diferentes factores técnicos, tácticos, físicos y psicológicos (Leo, Sánchez-Miguel, Sánchez-Oliva, Amado y García-Calvo, 2014). En este sentido, han aparecido trabajos sobre el fútbol, su historia y la cultura (García-Ocaña, 1994; FIFA, 2012; Martínez, 2001), la evolución del fútbol a lo largo de los mundiales (Castellano, Perea-Rodríguez y Hernández-Mendo, 2008) o las diferencias individuales de anticipación visual para interceptar penaltis en competición (Dicks, Davids y Button, 2010). También aparecieron trabajos que han estudiado la influencia de las variables tácticas y situacionales en las secuencias ofensivas durante los partidos de fútbol de élite (Sarmento et al., 2017), entre otros. Estos hechos han favorecido la proliferación de artículos de investigación sobre el deporte del fútbol en todos sus ámbitos (biomecánica, fisiología, psicología, técnica, táctica, etc.). Sin embargo, la mayoría de estos 
trabajos se centran mayoritariamente en temas relacionados con el resto de posiciones de los diferentes jugadores de campo, siendo escasos los estudios acerca del portero. En este sentido, es importante conocer la participación que tienen los porteros en los partidos para planificar su entrenamiento de acuerdo a la realidad competitiva. Para ello, es fundamental disponer de estudios e investigaciones que analicen la función que desempeña los porteros de un equipo de fútbol en competición.

\section{El portero de fútbol}

En el fútbol, el portero desempeña un puesto específico al ocupar un papel posicional único en el equipo dentro del terreno de juego (White et al., 2018). La posición del portero de fútbol ha sufrido una evolución importante en las últimas décadas. Además, en el fútbol moderno el portero debe responder a una exigencia técnico-táctica, en la organización colectiva del juego en ataque y en defensa. Por tanto, debe jugar con los pies muy a menudo y a dominar espacios entre la defensa y su portería para interceptar balones (Sainz de Baranda, Llopis y Ortega, 2005). Todo ello ha llevado consigo a que el portero de fútbol domine mejor los conceptos técnico-tácticos ofensivos y defensivos del juego. Como resultado de esto, además de las funciones defensivas, el guardameta debe tener un mayor protagonismo a nivel ofensivo en beneficio de un juego más dinámico y la organización de jugadas de ataque de manera premeditada (Sainz de Baranda et al., 2015).

En este sentido, los gestos técnico-tácticos son aspectos importantes que debe cumplir un guardameta, debido a que a través de estos, logra desarrollar sus habilidades y mejorar sus estrategias en conjunto. Por un lado, la técnica hace referencia a la capacidad de conseguir los resultados deseados utilizando un buen patrón de movimientos (Rebelo-Goncalves, Figueiredo, Coelho-e-Silva y Tessitore, 2016). De esta forma, actuando de manera eficiente, evitamos un gasto innecesario de la energía disponible durante la competición. Por otro lado, la táctica es la capacidad para aplicar correctamente los gestos técnicos, dependiendo en la situación en la que se encuentre en cada momento y respetando siempre las reglas de juego (Abellán, González Martí y FernándezBustos, 2009).Teniendo en cuenta ambas definiciones y el contexto real de juego, el entrenamiento más adecuado para el portero, no debe ser sólo el entrenamiento técnico y físico de forma individual, sino que será importante implementar dentro del entrenamiento los aspectos tácticos. Por tanto, el portero también debe estar integrado con sus compañeros de equipo y con orientaciones tanto ofensivas, como defensivas (Shamardin y Khorkavyy, 2015).

Según García-Ocaña (2008), las diferentes acciones técnico-tácticas que realiza un portero de fútbol en la competición se clasifican en: blocajes, interceptaciones, despejes o desvíos, salidas, lanzamientos, saques de puerta y otras. Teniendo en cuenta cada una de estas acciones técnico-tácticas, en el estudio realizado por Ruíz, Guerrero y Garrido (2007), comprobaron que las acciones que más realizaban los porteros de primera y segunda división española, eran los blocajes, los saques de puertas y los pases con el pie, con una media de 17,55 por partido. En la misma línea, los blocajes y los controles con el pie fueron las intervenciones que en más ocasiones realizaron los guardametas en el mundial 
de Corea y Japón en 2002 (Sainz de Baranda, Ortega y Palao, 2008). En este caso, los porteros realizaron una media de 25,2 acciones técnico-tácticas por partido. Estos resultados coinciden con los observados posteriormente por Muñoz, Muñoz, Cayetano, García y Muñoz (2016), en porteros profesionales durante la competición. Por otra parte, Sainz de Baranda y Ortega (2002) analizaron las acciones técnico-tácticas realizadas por los porteros en el mundial de Francia 98 y en la Eurocopa 2000. También, en la investigación realizada por Sainz de Baranda et al. (2019), analizaron las acciones ofensivas y defensivas de las porteras de fútbol en el campeonato del mundo de Alemania en 2011. Observaron que las porteras de los equipos que se clasificaron en fase de grupos, tuvieron un número superior de acciones ofensivas y realizadas con los pies, que las porteras de los equipos que quedaron eliminadas en la primera fase del campeonato. De igual manera, el estudio realizado por Hansen et al. (2017) con porteros de balonmano, observaron que los equipos que mejor clasificación obtuvieron en el campeonato del mundo de Dinamarca en 2015, sus porteros realizaron una intervención técnico-táctica más exitosa que aquellos porteros cuyos equipos finalizaron el campeonato en una peor clasificación.

Por otro lado, en el fútbol también se debe tener cuenta las diferencias existentes cuando se juega como local o como visitante. Desde una perspectiva general, Pollard (2008), comprobó que los equipos locales tenían una ventaja mayor para conseguir éxito en la competición. En la misma línea, Leite (2017), descubrió que jugar en casa aporta una ventaja para el resultado final de un partido. Un estudio realizado por Lago-Penas y Lago-Ballesteros (2011), concluyeron que los equipos que juegan como local tienen mayor probabilidad de meter goles y de tirar a portería. Por tanto, siguiendo estas investigaciones los porteros que juegan como visitantes tendrían más oportunidades de interactuar e intervenir en el juego durante la competición.

Cada uno de los resultados mostrados con anterioridad son importantes y se deben tener en cuenta cuando se trabaja con porteros. De esta manera, según Sainz de Baranda et al. (2005), con un análisis sobre la acción y participación del portero de fútbol, se podría aportar las claves para plantear tareas que aseguren un trabajo donde se desarrollen situaciones lo más parecidas a la lógica interna del fútbol. Para realizar un análisis completo sobre las acciones que realiza un portero en competición, la metodología observacional se caracteriza por poseer un alto rigor científico, ya que permite el estudio de comportamientos espontáneos sin influencia externa (Anguera, Camerino, Castañer, Sánchez-Algarra y Onwuegbuzie, 2017). Además, es uno de los recursos más importantes para mejorar el conocimiento del juego (Ardá, Maneiro, Rial, Losada y Casal, 2014). Por tanto, dicha información va a ser necesaria para su posterior aplicación en el entrenamiento y va a servir para determinar los contenidos prioritarios a trabajar en los entrenamientos específicos (Álvarez, 2012). En la actualidad, el objetivo a la hora de realizar entrenamientos con porteros es confeccionar un trabajo completo teniendo en consideración los aspectos técnicos, tácticos, físicos y psicológicos (Leo, Sánchez-Miguel, Sánchez-Oliva, Amado y García-Calvo, 2014). En esta línea, los ejercicios en el entrenamiento del portero tiene que ir orientado a mejorar la técnica (Ion, Serghei y Constantin, 2018) y los aspectos tácticos relacionados con las habilidades perceptivo- cognitivas (Rebelo-Goncalves et al., 2016). 
Además, es fundamental realizar actividades para trabajar la anticipación (Lidor, Ziv y Gershon, 2012), disminuyendo el tiempo de movimiento (Dicks, Uehara y Lima, 2011). Estos entrenamientos deben tener en cuenta una doble vertiente. Por un lado, trabajos específicos e individuales y por otro lado, junto a un trabajo colectivo o grupal. No obstante, el trabajo realizado en los entrenamientos tiene que ir orientado a las diferentes situaciones y acciones que se vayan a dar en cada partido.

Hasta el momento, solo se han analizado las acciones técnico-tácticas del portero en competiciones internacionales (Sainz de Baranda y Ortega, 2002), o en el fútbol base (Bezerra, Sabino, Ferreira y Vasconcellos, 2018). Sin embargo, desde nuestro conocimiento, no se han analizado las actuaciones en competición de los diferentes porteros pertenecientes a divisiones amateur y semi-profesionales. Además, tampoco se ha investigado sobre las diferencias existentes entre jugar como local o como visitante. Por lo tanto, el trabajo que se presenta a continuación, mediante el estudio de las acciones técnico-tácticas realizadas durante los partidos de fútbol en las diferentes divisiones, pretende aumentar el conocimiento existente sobre el portero de fútbol y su trabajo realizado en el ámbito competitivo.

\section{OBJETIVOS}

Los objetivos de la investigación fueron: 1) Cuantificar el número de acciones técnico-tácticas totales por partido que realiza el portero de fútbol dentro de la competición y analizar las diferencias entre las divisiones (primera, segunda, segunda B y tercera); 2) analizar si existen diferencias entre cada una de las divisiones estudiadas en cuanto al número y tipo de acciones técnico-tácticas llevadas a cabo por el portero de fútbol y 3) examinar las diferencias entre el tipo y número de acciones técnico-tácticas que se realizan cuando se juega como local o como visitante.

\section{MATERIAL Y MÉTODOS}

\section{PARTICIPANTES}

La muestra poblacional sometida a análisis fue de 160 porteros (40 porteros de cada división). Las divisiones analizadas han sido las siguientes: primera división, segunda división, segunda división $\mathrm{B}$ y tercera división. En relación a las divisiones analizadas, en segunda división B, se seleccionó el Grupo IV, formado por clubes de las Comunidades Autónomas de Murcia, Castilla La Mancha, Andalucía y Extremadura. De los 18 grupos pertenecientes a la tercera división, se escogió al Grupo XIV, formado por 20 clubes extremeños. En la Tabla 1 se describen la edad y las características antropométricas de los porteros sometidos a estudio: 
Tabla 1. Características antropométricas de los porteros de fútbol.

\begin{tabular}{|c|c|c|c|c|c|}
\hline \multicolumn{6}{|c|}{ DIVISIÓN } \\
\hline & Primera & Segunda & Segunda & Tercera & \multirow{3}{*}{$\chi^{2}$} \\
\hline & $\begin{array}{l}\text { División } \\
(n=40)\end{array}$ & $\begin{array}{l}\text { División } \\
(n=40)\end{array}$ & $\begin{array}{l}\text { División B } \\
(n=40)\end{array}$ & $\begin{array}{l}\text { División } \\
(n=40)\end{array}$ & \\
\hline & $M(D T)$ & $M(D T)$ & $M(D T)$ & $M(D T)$ & \\
\hline $\begin{array}{l}\text { Edad } \\
\text { (años) }\end{array}$ & $29,63(3,87)$ & $29,47(4,56)$ & $28,03(4,63)$ & $27,30(4,89)$ & 5,39 \\
\hline Peso (kg) & $86,23(19,36)^{a}$ & $80,77(3,61)^{a b}$ & $79,53(5,36)^{b}$ & $79,00(5,94)^{b}$ & $11,90^{* *}$ \\
\hline Altura $(\mathrm{cm})$ & $184,03(20,02)^{a}$ & $186,80(4,96)^{a b}$ & $184,97(3,35)^{b}$ & $179,93(3,35)^{b}$ & $12,53^{*}$ \\
\hline
\end{tabular}

\section{INSTRUMENTOS}

Para la cuantificación del número de acciones técnico-tácticas realizadas durante un partido de fútbol por parte de los porteros, se utilizó la metodología observacional, por ser una estrategia útil para mejorar la comprensión y el conocimiento real del juego (Mombaerts, 2000). Dicho método de análisis va a permitir la valoración de la actividad del portero en toda su complejidad, respetando su carácter interactivo y su propio contexto, la competición y garantizando la no intervención directa en el proceso de observación (Anguera, Portell, Chacón-Moscoso y Sanduvete-Chaves, 2018).

Siguiendo la guía de García-Ocaña (2008) sobre las diferentes acciones técnicotácticas del portero de fútbol, para esta investigación se han analizado y cuantificado las siguientes: blocaje, desvío, despeje, otros despejes, rechace, control, conducción, regate, pase, saque de puerta, saque de tiro libre y salidas (1X1 y cobertura táctica). En cada una de dichas acciones, siguiendo a Ibarrola (2011), se ha tenido en cuenta la altura de ejecución (alta, media y baja), si se hizo con o sin salto y si dicho salto fue lateral o vertical. Para la recopilación de estos datos, se utilizó la "Ficha de Observaciones" que aparece en la Tabla 2. Esta ficha fue dividida en seis columnas, de más general a más específico (de izquierda a derecha). Para la presente investigación, para el análisis de las acciones técnico-tácticas se han diferenciado entre acciones medias realizadas por partido, las acciones técnico-tácticas grupales y las técnico-tácticas específicas. Para un análisis más general sobre las acciones técnico-tácticas grupales, se ha utilizado solamente la columna de acción, observada en la Tabla 2. Sin embargo, para las acciones técnico-tácticas específicas se tuvo en cuenta la altura del móvil, si la acción se realizó con o sin salto y si dicho salto se efectuó de forma lateral (horizontal) o vertical. Por tanto, para el análisis de las acciones específicas, se tuvieron en cuenta cada una de las variantes de las cuatro primeras columnas presentes en la Tabla 2. 
Rev.int.med.cienc.act.fís.deporte - vol. 20 - número 80 - ISSN: 1577-0354

Tabla 2. Hoja de observación de las diferentes acciones técnico-tácticas de los porteros de fútbol en competición

\begin{tabular}{|c|c|c|c|c|c|}
\hline ACCIÓN & $\begin{array}{l}\text { ALTURA/ } \\
\text { LONGITUD }\end{array}$ & SALTO & FORMA & LOCAL & VISITANTE \\
\hline \multirow{8}{*}{ BLOCAJE } & \multirow{3}{*}{ Alto } & \multirow{2}{*}{ Con salto } & Lateral & & \\
\hline & & & Vertical & & \\
\hline & & Sin salto & & & \\
\hline & \multirow{3}{*}{ Medio } & \multirow{2}{*}{ Con salto } & Lateral & & \\
\hline & & & Vertical & & \\
\hline & & Sin salto & & & \\
\hline & \multirow{2}{*}{ Bajo } & Con salto & Lateral & & \\
\hline & & Sin salto & & & \\
\hline \multirow{9}{*}{ DESVío } & \multirow{3}{*}{ Alto } & \multirow{2}{*}{ Con salto } & Lateral & & \\
\hline & & & Vertical & & \\
\hline & & Sin salto & & & \\
\hline & \multirow{3}{*}{ Medio } & \multirow{2}{*}{ Con salto } & Lateral & & \\
\hline & & & Vertical & & \\
\hline & & Sin salto & & & \\
\hline & \multirow{2}{*}{ Bajo } & Con salto & Lateral & & \\
\hline & & Sin salto & Vertical & & \\
\hline & Mano cambiada & Suele ser con salto & & & \\
\hline \multirow{9}{*}{ DESPEJE } & \multirow{3}{*}{ Alto } & \multirow{2}{*}{ Con salto } & Lateral & & \\
\hline & & & Vertical & & \\
\hline & & Sin salto & & & \\
\hline & & & Lateral & & \\
\hline & Medio & Con salto & Vertical & & \\
\hline & & Sin salto & & & \\
\hline & & Con salto & Lateral & & \\
\hline & Bajo & Sin salto & & & \\
\hline & Mano cambiada & Suele ser con salto & & & \\
\hline & Pнг̃ & Con salto & & & \\
\hline DESPEJE & Puño & Sin salto & & & \\
\hline (OTROS) & Pie & & & & \\
\hline & Cabeza & Suele ser con salto & & & \\
\hline RECHACE & Tras salida & & & & \\
\hline CONTROI & Pie & & & & \\
\hline CONTROL & Pecho & & & & \\
\hline CONDUCCIÓN & & & & & \\
\hline REGATE & & & & & \\
\hline & Mano & Largo & & & \\
\hline PASF & Mano & Corto & & & \\
\hline & Pie & Largo & & & \\
\hline & Pie & Corto & & & \\
\hline SAQUE DE & & Largo & & & \\
\hline PUERTA & & Corto & & & \\
\hline SAQUE TIRO & & Largo & & & \\
\hline LIBRE & & Corto & & & \\
\hline & $1 \times 1$ & & & & \\
\hline & Despejar & & & & \\
\hline SALIDAS & Pasar & & & & \\
\hline & Cobertura táctica & & & & \\
\hline
\end{tabular}




\section{PROCEDIMIENTO}

La investigación fue aprobada por la Comisión Ética de la Universidad, cumpliendo las normas establecidas en la Declaración de Helsinki de 1964 y con las exigencias éticas que marca la American Psychological Association (APA, 2009). Se ha llevado a cabo el visionado de 80 partidos en la temporada 20162017 de las cuatro divisiones más importantes del fútbol español: 20 partidos fueron de primera división, 20 de segunda división, 20 de segunda división $B$ y 20 de tercera división, con un total de 160 porteros analizados. La observación se realizó mediante un diseño nomotético, de seguimiento y multidimensional (Anguera, Blanco-Villaseñor, Hernández-Mendo, y Losada, 2011).

El visionado de los partidos se llevó a cabo por un observador. Para asegurar la validez y fiabilidad del observador, antes de comenzar con la investigación se realizó un programa de entrenamiento inter e intra observadores. Para este proceso de formación se tuvieron como referencia las fases de entrenamiento apoyadas por Anguera y Hernández-Mendo (2013) y de acuerdo con los objetivos específicos de la presente investigación. El programa inter observadores se realizó con diferentes profesionales del entrenamiento del portero. Para ello, todos los participantes del entrenamiento, observaron de forma individual los mismos partidos de cada división incluida en el presente estudio. Posteriormente, cuando un grupo de expertos realizó un análisis de contenido para examinar la concordancia entre las diferentes observaciones realizadas, se comenzó con el programa de entrenamiento intra observador. En este caso, el investigador responsable del visionado de todos los partidos de la presente investigación, observó en 2 ocasiones varios partidos de todas las divisiones analizadas. Se compararon los resultados entre las 2 observaciones realizadas de los partidos seleccionados y se comparó los resultados obtenidos entre el visionado directo e indirecto de las acciones técnico-tácticas en competición. De igual manera, el grupo de expertos examinó de nuevo la concordancia entre las diferentes observaciones realizadas.

Por otro lado, con el fin de conseguir unos resultados objetivos y aumentar la variabilidad de análisis, la observación se realizó de manera intencionada. Se analizaron partidos tanto de la primera como de la segunda vuelta. También, se tuvo en cuenta que al menos en una ocasión, se observaran a todos los equipos que componen cada división. Por otra parte, se tuvo en cuenta que las características morfológicas y dimensionales de los terrenos de juego fueran diferentes en cada uno de los partidos analizados. Por último, se analizaron las acciones técnico-tácticas de los porteros en diferentes condiciones meteorológicas. En este caso, se seleccionaron aquellos partidos que mostraron diferentes condiciones atmosféricas, tales como la temperatura, lluvia, sol, aire, etc.

Por último, el proceso de observación de los partidos seleccionados se realizó siguiendo en todo momento unas pautas precisas. Se observó un solo partido al día y en horario de mañana. Además, todos los partidos se observaron en la misma sala, manteniendo una buena iluminación y un silencio preciso para mantener la concentración. 


\section{ANÁLISIS ESTADÍSTICO}

Para el análisis de los datos se empleó el paquete estadístico SPSS 19. Se empleó una prueba de Kolmogorov-Smirnov para evaluar la normalidad de los datos. La prueba de Rachas para determinar la aleatoriedad y para comprobar la homoscedasticidad de la muestra, se aplicó la prueba de Levene. De acuerdo con estas pruebas y a los resultados obtenidos, se determinó un análisis no paramétrico y de muestras independientes entre sí. Seguidamente, se utilizó la prueba $\mathrm{H}$ de Kruskal-Wallis para analizar las diferencias en las acciones técnicotácticas llevadas a cabo por los porteros durante los partidos de fútbol en las diferentes divisiones de fútbol (con un nivel de significación de $p \leq 0,05$ ). Por otro lado, se utilizó la prueba $U$ de Mann- Whitney para analizar la incidencia que tenía el jugar como local o como visitante, en relación a las acciones técnicotácticas totales realizadas por los porteros de fútbol en los partidos de competición.

\section{RESULTADOS}

\section{ACCIONES TÉCNICO-TÁCTICAS MEDIAS POR PARTIDO}

A continuación, se describen el número de acciones técnico-tácticas medias por partido, que han realizaron los porteros de cada una de las divisiones y en cada partido observado.

Los porteros de primera división realizaron un total de 51,87 acciones técnicotácticas por partido durante la competición. En el caso de los porteros de segunda división, han realizado de media 49,74 actuaciones. En la segunda división $B$ las acciones técnico-tácticas medias totales fueron de 46,83, y, por último, los porteros de tercera división realizaron 45,62 intervenciones técnicotácticas totales por partido. Sin embargo, tras la aplicación de las pruebas estadísticas, los resultados no mostraron diferencias significativas $(p<0,05)$ sobre el número total de gestos técnico-tácticos realizados por los porteros entre las divisiones analizadas.

\section{ACCIONES TÉCNICO-TÁCTICAS GRUPALES}

En la Tabla 3, se muestran el número de acciones técnico-tácticas grupales llevadas a cabo en la competición por el portero de fútbol, separados por divisiones. 
Tabla 3. Efecto de las diferentes divisiones (primera, segunda, segunda B y tercera) sobre el número de acciones técnico-tácticas grupales llevadas a cabo por los porteros de fútbol durante un partido.

\begin{tabular}{|c|c|c|c|c|c|}
\hline \multicolumn{6}{|c|}{ DIVISIÓN } \\
\hline & $\begin{array}{l}\text { Primera } \\
\text { División } \\
(n=20)\end{array}$ & $\begin{array}{l}\text { Segunda } \\
\text { División } \\
(n=20)\end{array}$ & $\begin{array}{c}\text { Segunda } \\
\text { División B } \\
(n=20)\end{array}$ & $\begin{array}{l}\text { Tercera } \\
\text { División } \\
(n=20)\end{array}$ & $\chi^{2}$ \\
\hline & $M(D T)$ & $M(D T)$ & $M(D T)$ & $M(D T)$ & \\
\hline Blocajes & $5,72(3,23)^{b}$ & $7,92(2,56)^{\mathrm{a}}$ & $7,59(4,57)^{\mathrm{ab}}$ & $9,19(3,39)^{a}$ & $20,94^{\star \star \star}$ \\
\hline Desvíos & $0,62(0,63)$ & $0,42(0,59)$ & $0,59(0,94)$ & $0,45(0,67)$ & 2,46 \\
\hline Despejes & $1,18(1,42)$ & $0,92(0,91)$ & $0,83(0,75)$ & $0,71(0,90)$ & 3,72 \\
\hline Otros despejes & $2,79(1,67)$ & $2,82(1,85)$ & $2,59(1,57)$ & $2,45(1,71)$ & 1,00 \\
\hline Rechaces & $0,41(0,54)$ & $0,44(0,72)$ & $0,34(0,61)$ & $0,77(0,88)$ & 5,95 \\
\hline Controles & $10,92(7,02)^{\mathrm{a}}$ & $7,47(3,95)^{\mathrm{b}}$ & $5,66(3,08)^{b c}$ & $4,45(3,01)^{c}$ & $30,07^{* * *}$ \\
\hline Pases mano & $3,46(2,18)^{a b}$ & $3,82(2,19)^{\mathrm{a}}$ & $2,38(2,09)^{b}$ & $3,10(2,00)^{\mathrm{ab}}$ & $9,71^{*}$ \\
\hline Saques de puerta & $9,90(5,38)^{b}$ & $10,92(2,79)^{\mathrm{ab}}$ & $13,41(4,84)^{\mathrm{a}}$ & $11,94(4,96)^{\mathrm{ab}}$ & $11,26^{*}$ \\
\hline Salidas & $1,82(2,05)$ & $2,68(1,93)$ & $2,24(1,66)$ & $1,77(1,45)$ & 6,01 \\
\hline
\end{tabular}

Nota. ${ }^{*} p<0,05,{ }^{* *} p<0,01,{ }^{\star *} p<0,001$. Los resultados del análisis de la prueba $\mathrm{H}$ de Krustal- Wallis están indicados con los subcriptores ${ }^{a},{ }^{b} y^{c}$, los cuales, reflejan las diferencias significativas entre cada una de las divisiones.

Como se puede observar, los tres gestos técnico-tácticos más utilizados son los controles, seguidos por los saques de puerta y los blocajes, respectivamente. Los gestos que menos han realizado los porteros de fútbol han sido los desvíos, despejes y los rechaces.

\section{ACCIONES TÉCNICO-TÁCTICAS ESPECÍFICAS}

En la Tabla 4, se muestran las medias de todas las acciones técnico-tácticas específicas en las que se obtuvieron diferencias significativas teniendo en cuenta cada una de las divisiones (primera, segunda, segunda B y tercera). 
Tabla 4. Diferencias en el número de acciones técnico-tácticas específicas llevadas a cabo por los porteros de fútbol de las diferentes divisiones (primera, segunda, segunda B y tercera)

\begin{tabular}{|c|c|c|c|c|c|}
\hline \multicolumn{6}{|c|}{ DIVISIÓN } \\
\hline & $\begin{array}{l}\text { Primera } \\
\text { División } \\
(n=20)\end{array}$ & $\begin{array}{c}\text { Segunda } \\
\text { División } \\
(n=20)\end{array}$ & $\begin{array}{c}\text { Segunda } \\
\text { División B } \\
(n=20)\end{array}$ & $\begin{array}{l}\text { Tercera } \\
\text { División } \\
(n=20)\end{array}$ & $\chi^{2}$ \\
\hline & $M(D T)$ & $M(D T)$ & $M(D T)$ & $M(D T)$ & \\
\hline Blocaje alto con salto vertical & $0,83(0,90)^{\mathrm{a}}$ & $1,05(0,85)^{\mathrm{a}}$ & $1,23(1,04)^{\mathrm{ab}}$ & $1,82(1,03)^{b}$ & $18,70^{* * *}$ \\
\hline Blocaje medio sin salto & $1,60(1,55)^{b}$ & $2,88(1,80)^{\mathrm{a}}$ & $2,77(1,77)^{\mathrm{a}}$ & $3,62(1,95)^{a}$ & $24,54^{* * *}$ \\
\hline Control con pie & $11,03(7,11)^{\mathrm{a}}$ & $7,28(3,92)^{\mathrm{ab}}$ & $5,33(3,26)^{\mathrm{bc}}$ & $4,15(2,95)^{c}$ & $33,44^{* \star *}$ \\
\hline Desvío alto con salto lateral & $0,03(0,16)^{b}$ & $0,05(0,22)^{\underline{a}}$ & $0,17(0,38)^{\mathrm{a}}$ & $0,21(0,41)^{b}$ & $8,90^{*}$ \\
\hline Pase con mano en corto & $2,83(1,93)^{\mathrm{ab}}$ & $3,00(2,12)^{\mathrm{a}}$ & $1,77(1,55)^{b}$ & $2,15(1,65)^{\mathrm{ab}}$ & $8,95^{\star}$ \\
\hline Pase con pie en corto & $7,18(6,54)^{a}$ & $3,98(3,21)^{a b}$ & $3,27(3,38)^{b}$ & $2,15(2,23)^{b}$ & $16,84^{\star *}$ \\
\hline Salidas con $1 \times 1$ & $0,02(0,98)^{\mathrm{ab}}$ & $0,00(0,00)^{\mathrm{a}}$ & $0,03(0,19)^{\mathrm{ab}}$ & $0,22(0,42)^{b}$ & $11,66^{*}$ \\
\hline Salida y cobertura táctica & $1,23(1,12)^{\mathrm{bc}}$ & $2,03(1,39)^{a}$ & $1,83(1,39)^{\mathrm{ab}}$ & $1,12(0,77)^{\mathrm{c}}$ & $11,77^{\star *}$ \\
\hline Saque de puerta en largo & $4,40(2,89)^{\mathrm{b}}$ & $6,65(2,79)^{a}$ & $8,60(4,37)^{\mathrm{a}}$ & $7,18(3,90)^{\mathrm{a}}$ & $21,21^{* *}$ \\
\hline
\end{tabular}

Nota. ${ }^{*} p<0,05,{ }^{* *} p<0,01,{ }^{* * *} p<0,001$. Los resultados del análisis de la prueba $\mathrm{H}$ de Krustal- Wallis están indicados con los subcriptores ${ }^{a},{ }^{b} y^{c}$, los cuales, reflejan las diferencias significativas entre cada una de las divisiones.

Como se puede observar, de las 48 acciones técnico-tácticas específicas estudiadas en cada división, 9 de ellas mostraron diferencias significativas $(p<$ $0,05)$. En el caso del blocaje alto con salto vertical, el mayor valor fue observado en tercera división no habiéndose encontrado diferencia entre el resto de divisiones. En línea con el gesto técnico-táctico anterior, el blocaje medio sin salto mostró un valor inferior en primera división. En el caso del CPIE, el mayor valor se dio en primera división con respecto a las demás divisiones estudiadas. Dicho gesto técnico-táctico va disminuyendo en el número de realizaciones a medida que descendemos de categoría.

Por otro lado, se observó que el número de desvío alto con salto lateral fueron menores en primera división, y a simple vista, se llevaron a cabo en muchas menos ocasiones con respecto a otras acciones técnico-tácticas. Con respecto a los pases con mano en corto fue en la segunda división B la que presentó un valor menor que la segunda división, pero sin mostrar diferencias significativas con respecto a primera o tercera división. Sin embargo, los porteros de primera división llevaron a cabo un mayor número del pase con pie en corto que el resto de divisiones.

En cuanto a la acción técnico-táctica salida y cobertura táctica, fue llevada a cabo en menores ocasiones por los porteros de tercera división. Por último, con respecto a los saques de puerta en largo, los porteros de primera división la realizaron en menor número de ocasiones. 


\section{DIFERENCIAS DE ACCIONES TÉCNICO-TÁCTICAS LOCALES Y VISITANTES}

La presente tabla, muestra las medias de todas las acciones técnico-tácticas que obtuvieron diferencias significativas, realizadas por los porteros estudiados, dependiendo de si jugaban como local o como visitantes, en todas las divisiones estudiadas (primera, segunda, segunda B y tercera).

Tabla 5. Acciones totales que mostraron diferencias significativas entre los porteros locales y visitantes.

\begin{tabular}{lccc}
\hline & $\begin{array}{c}\text { LOCAL } \\
(n=80)\end{array}$ & $\begin{array}{c}\text { VISITANTE } \\
(n=80)\end{array}$ & \multirow{2}{*}{$\chi 2$} \\
\cline { 1 - 3 } & $M(D T)$ & $M(D T)$ & \\
\hline Desvío alto con salto lateral & $0,06(0,23)$ & $0,15(0,36)$ & $2,44^{*}$ \\
Despejes bajos con salto lateral & $0,18(0,45)$ & $1,38(0,61)$ & $2,01^{*}$ \\
Saque de puerta en largo & $6,24(3,90)$ & $6,88(3,58)$ & $1,99^{*}$ \\
Salidas con uno contra uno & $0,04(0,20)$ & $0,21(0,77)$ & $1,96^{*}$ \\
\hline
\end{tabular}

Nota. ${ }^{*} p<0,05$ : según Prueba $U$ de Mann-Whitney

Teniendo como factor fijo las variables de "local" y "visitante", a la hora de realizar el estudio y análisis de datos, se observó que, de 48 acciones totales estudiadas dicho factor sólo tuvo efecto significativo sobre 4 de ellas, como se muestra en la Tabla 5.

Atendiendo a dichos resultados, se observa que los porteros visitantes tienen un número mayor de despejes con respecto a los porteros locales. Además, son los porteros visitantes los que realizan la acción de salidas con $1 \times 1$ un número mayor de veces, durante un partido. Por lo tanto, los porteros visitantes intervienen en este tipo de gestos técnico-tácticos determinantes, en un número mayor de ocasiones.

Por otro lado, teniendo en cuenta la acción de saque de puerta en largo, los porteros visitantes la realizan un valor mayor de acciones técnico-tácticas que los porteros que jugaban como local.

\section{DISCUSIÓN}

El desarrollo de este estudio trataba de aportar nuevo conocimiento sobre el número de acciones técnico-tácticas realizadas por el portero de fútbol en diferentes divisiones y averiguar si existían diferencias en el número y tipo de éstas acciones en función de la división o en función de si jugaba como local o como visitante. En líneas generales, se puede observar en los resultados, que los porteros de las diferentes divisiones realizan un número similar de acciones ténicos-tácticas, pero que el tipo de acciones que realiza parece variar en función de la división de la que juega y si lo hace como local o visitante.

De forma específica, el primer objetivo del estudio fue cuantificar el número de las acciones técnico-tácticas totales por partido que realiza el portero de fútbol dentro de la competición entre las diferentes divisiones analizadas. Según los resultados obtenidos, no se han encontrado diferencias significativas entre las 
divisiones estudiadas. Por tanto, se podría afirmar que la variabilidad del número de acciones mostradas en cada división no está relacionada con la propia actuación del portero durante la propia competición. Estas diferencias pueden estar relacionadas con el estilo de juego característico de los equipos cuando juegan como local y como visitante (Muñoz et al., 2016), o por las difencias existentes entre la parte ofensiva y defensiva, entre otras. Además, puede ser debido a un mayor número de finalización del equipo contrario al realizar un mayor número de contraataques (Tenga, Holme, Ronglan y Bahr, 2010). Otra de las características que hace que un portero realice un mayor o menor número de intervenciones, es la posición que ocupa su equipo en la tabla clasificatoria (Hansen et al., 2017).

El segundo objetivo de la investigación fue analizar las diferencias entre cada una de las divisiones estudiadas en cuanto al número y tipo de acciones técnicotácticas llevadas a cabo por el portero de fútbol en competición. Teniendo en cuenta los resultados obtenidos sobre los gestos técnico-tácticos grupales, tiene relación con el estudio de Vales, Sambade y Areces (2002), donde expone que los gestos más utilizados son los saques de puerta, los pases con el pie y los blocajes. De igual manera, estos tres gestos técnico-tácticos fueron los más realizados por los porteros de primera y segunda división en la temporada 20042005 (Ruíz et al., 2007). En la misma línea, el estudio que realizaron los autores Vales et al. (2002), concuerda con los resultados obtenido en la presente investigación en relación a la acción técnico-táctica del rechace, siendo una de las menos utilizadas por los porteros en competición. Sin embargo, Vales et al. (2002) sólo estudiaron a los porteros que compiten en la élite y no los pertenecientes a ninguna categoría semi-profesional. Además, dicho estudio tampoco describió los gestos técnico-tácticos de una manera específica, ni tuvieron en cuenta si fueron realizados con salto o no, la altura de la pelota o la forma de realizar la acción. Es por ello, que sólo podría compararse con los resultados obtenidos de los porteros de la primera división.

En cuando a las acciones técnico-tácticas específicas estudiadas, el blocaje alto con salto vertical fue observado en un mayor número de veces, en la tercera división. Esto puede ser debido a un mayor número de lanzamientos sin éxito desde media o larga distancia a zonas centrales de la portería. En la misma línea que la acción anterior, el blocaje medio sin salto mostró un valor inferior en primera división. Con estos datos, la afirmación de que para tener un mayor éxito de cara a portería está directamente relacionada con la calidad y no con la cantidad, cobra más veracidad. En el caso del control con pie, va disminuyendo en el número de realizaciones a medida que descendemos de categoría. Un hecho que puede estar relacionado con este resultado es el estado del césped de los terrenos de juego, sobre todo en aquellos partidos donde las condiciones meteorológicas fueron adversas (lluvia, frío, etc.), viéndose dificultada la acción de este gesto técnico-táctico en los porteros. De hecho, en los campos de primera y segunda división el césped y sus cuidados son bastante mejores que en segunda división B y tercera división, respectivamente. Por otro lado, este tipo de comportamientos técnico-tácticos, puede servir de gran ayuda a entrenadores para planificar y plantear diferentes acciones tácticas durante la competición (Sampaio y Maçãs, 2012). Relacionando el gesto técnico-táctico del control con pie con otros estudios, los porteros de la Premier League de Ucrania realizaron 
un número menor de ellos durante la competición (Shamardin y Khorkavyy, 2015). De la misma forma, comparándolo con el estudio de Sainz de Baranda et al. (2008), los porteros del mundial de Corea y Japón en 2002 realizaron menos controles que los porteros de primera y segunda división del presente estudio. Sin embargo, si lo comparamos con los controles con pie realizados por los porteros de segunda b y tercera división, realizaron un número menor de ellos.

Por otro lado, el desvío alto con salto lateral se observó en un menor número de ocasiones con respecto a los gestos técnico-tácticos anteriores. Sin embargo, los pases con pie en corto representan valores mayores en todas las divisiones, sobre todo en la primera división, donde se observa un mayor número de pases con pie en corto. Este resultado no concuerda el estudio realizado por Sainz de Baranda et al. (2019), donde las porteras realizaron un número mayor de pases en largo durante el campeonato del mundo de 2011. Las diferencias observadas en la acción de salida y cobertura táctica, puede ser debida a una menor distancia entre el portero y la primera línea defensiva en tercera división. Para finalizar con las acciones técnico-tácticas específicas, con respecto al saque de puerta en largo, los resultados evidencian, que el juego llevado a cabo por los jugadores de la primera división española, se basa en acciones técnico-tácticas fundamentalmente realizadas en corto. Estos resultados concuerdan con la investigación de Muñoz et al. (2016), no obstante, el número de acciones de saques de puerta en largo realizadas por los porteros profesionales, fue menor que las efectuadas por los porteros analizados en el presente trabajo. De esta forma, en el fútbol profesional se elabora un juego más combinativo y no tan directo, como se puede observar en segunda división B y tercera. En estas dos categorías, predomina los pases largos y el juego más directo desde zonas de iniciación o desde los sectores defensivos hasta zonas de finalización y cercanas a la portería contraria. Además, estas diferencias en el juego, puede ser debido al status del partido o al momento de la temporada en el que se esté compitiendo (Taylor, Mellalieu, James y Barter, 2010).

Por último, el tercer objetivo fue examinar las diferencias entre el tipo y número de las acciones técnico-tácticas que se realizan cuando se juega como local o como visitante. En este caso, se observa que los guardametas visitantes intervienen en acciones técnico-tácticas determinantes, en un número mayor de ocasiones. Dentro de la realidad competitiva, a causa del ambiente competitivo, es más difícil tener una buena comunicación entre jugadores, en el equipo visitante (Boyko, Boyko y Boyko, 2007). No obstante, las cuatro acciones que mostraron diferencias significativas, el número de acciones que se realizan por partidos son bastante reducidas. Sin embargo, según Sainz de Baranda et al. (2008), son gestos técnico-tácticos que aunque se realizan en muy pocas ocasiones durante la competición, son acciones claves en un partido y que deben tenerse en cuenta. Además, considerando que las diferencias existentes entre las acciones locales y visitantes son mínimas, se puede decir que los equipos locales tienen mayor probabilidad de conseguir mayores resultados positivos que el contrario, ya que en cuanto al número de acciones, es superior el del portero contrario (Muñoz et al., 2016). Por otro lado, los resultados en relación a la acción de saque de puerta en largo, coinciden con el estudio realizado por Muñoz et al. (2016). Según estos autores los resultados reflejan que cuando un equipo juega en su propio campo, desde el portero, intenta realizar un juego más elaborado y 
combinativo. Por el contrario, el inicio del juego de los equipos visitantes, se basa más en un juego directo y en largo. Es importante destacar la gran variedad de acciones técnico-tácticas que realiza un portero de fútbol durante la competición (García-Ocaña, 2008). Por tanto, son de gran utilidad y deben tenerse en cuenta, a la hora de realizar la planificación semanal. Dicha planificación debe estar enmarcada en un contexto específico y confeccionada junto a todos los miembros del cuerpo técnico. De esta manera, se trabajará de manera más específica e individualizada con cada uno de los porteros del equipo.

\section{CONCLUSIONES, LIMITACIONES Y PROPUESTAS DE FUTURO}

El conjunto de datos registrados y analizados en este estudio sobre las exigencias técnico-tácticas a las que están sometidos los porteros de fútbol dependiendo de la división en la que compitan, se han extraído las siguientes conclusiones: 1) Al no encontrar diferencias significativas en las acciones técnico-tácticas totales de los porteros, se podría decir que los aspectos diferenciables e influenciables en la participación total de un portero de fútbol, se deben a factores externos al portero y a su actuación durante la competición. Por tanto, a la hora de diseñar el número de acciones técnico-tácticas medias por entrenamientos, este tipo de planificación no debe variar entre las divisiones analizadas; 2) La división en la que juega el portero tiene un efecto significativo sobre 9 acciones técnico-tácticas específicas. Además, el gesto técnico-táctico que más han realizado los porteros de las 4 divisiones estudiadas y empleados principalmente por la acción del tren superior, ha sido el blocaje. También, se ha observado que el dominio del juego con los pies en el portero de fútbol actual es fundamental, independientemente de la división en la que se encuentre; 3) Hay que tener en cuenta en la planificación técnico-táctica de un portero de fútbol, el lugar donde se va a celebrar la próxima competición, ya que se atisban pequeñas diferencias en cuanto al número de acciones en ambos escenarios.

Según los datos obtenidos en este estudio, el conocimiento de las acciones técnico-tácticas suponen un paso muy importante en el análisis de la práctica competitiva del portero de fútbol, ya sea a nivel específico (individual) o colectivo (junto con todo el equipo). Por tanto sirve de ayuda a profesionales del sector de cada una de las divisiones analizadas y a realizar una planificación del entrenamiento, contextualizada con la realidad competitiva del portero de fútbol.

Teniendo en cuenta el apartado anterior, los profesionales encargados de la planificación de los porteros, deben prestar atención al aprendizaje y perfeccionamiento del blocaje en todas sus formas. La especialización de este gesto técnico-táctico es fundamental para conseguir resultados óptimos y positivos en la competición, ya que es un gesto que se repite durante la competición y que al realizarlo correctamente, evitar segundas jugadas del contrario y permitir tener la posesión del balón. Además, es imprescindible iniciar al portero en el juego de pies desde edades tempranas. Para ello, es fundamental introducirlo dentro de los ejercicios y entrenamientos con todo el grupo, donde se realicen frecuentemente este tipo de acciones técnico-tácticas dentro de un entorno competitivo. 
Por otro lado, aunque existes gestos técnico-tácticos que rara vez se producen en la competición, son acciones muy determinantes en el resultado final de un partido y que los especialistas de porteros deberían tenerlo en cuenta durante toda la temporada, para conseguir mejores resultados.

Por lo tanto, la máxima intención con esta propuesta es la de abordar el entrenamiento del portero de fútbol, de una manera global insistiendo en la integración de todos los conceptos técnico-tácticos. También se pretende conseguir una mejora en los entrenamientos específicos llevados a cabo con los porteros de fútbol, en relación a lo investigado en el presente estudio y dependiendo en la división en la que se encuentren.

En relación a las limitaciones del estudio, por un lado, no se tuvieron en cuenta sí las diferentes acciones técnico-tácticas se realizaron correctamente y se obtuvo un rendimiento en el juego. Por otro lado, tampoco se tuvo en cuenta si las diferentes acciones fueron ofensivas o defensivas. En esta línea, otro problema encontrado a la hora de evaluar los gestos técnico-tácticos ha sido la diversidad y heterogeneidad en cuanto a las hojas e instrumentos de observación, puesto que cada autor ha utilizado una terminología y orden específico.

Sería interesante continuar investigando en esta línea, ampliando la muestra de partidos analizados, introduciendo en dicha investigación a otros grupos de tercera y segunda división B, para homogeneizar estas dos categorías. También otro de los objetivos futuros para investigar, es comparar el fútbol femenino con el masculino dentro del ámbito nacional. Además, también sería interesante analizar las acciones técnico-tácticas de los porteros que compiten en otras grandes ligas europeas y de esta manera comparar los resultados con los obtenidos con nuestro presente estudio. Por último, sería útil analizar el rendimiento de las acciones realizadas por los porteros de cada división, durante la competición.

\section{REFERENCIAS BIBLIOGRÁFICAS}

Abellán, J., González-Martí, I., y Fernández-Bustos, G. (2009). V Congreso nacional de las ciencias del deporte y la educación física. E.U. Magisterio Albacete. Universidad de Castilla La Mancha, ISBN: 978-84-613-1660-1

Álvarez, J. (2012). La actividad competitiva del portero de fútbol: análisis objetivo y orientaciones para el entrenamiento específico. Revista de Preparación Física en el Fútbol, 3, 69-83.

Anguera, M.T., Blanco-Villaseñor, A., Hernández-Mendo, A., y Losada, J.L. (2011). Diseños observacionales: ajuste y aplicación en psicología del deporte. Cuadernos de Psicología del Deporte, 11, 63-76.

Anguera, M. T., Camerino, O., Castañer, M., Sánchez-Algarra, P., y Onwuegbuzie, A. J. (2017). The specificity of observational studies in physical activity and sports sciences: Moving forward in mixed methods research and proposals for achieving quantitative and qualitative symmetry. Frontiers in psychology. 8, 2196. doi:10.3389/fpsyg.2017.02196

Anguera, M. T., y Hernández-Mendo, A. (2013). La metodología observacional en el ámbito del deporte. E-Balonmano: Revista de Ciencias del Deporte, 9, 135-160. 
Anguera, M. T., Portell, M., Chacón-Moscoso, S., y Sanduvete-Chaves, S. (2018). Indirect observation in everyday contexts: Concepts and methodological guidelines within a mixed methods framework. Frontiers in psychology, 9, 13. doi:10.3389/fpsyg.2018.00013

Ardá, T., Maneiro, R., Rial, A., Losada, J. L., y Casal, C.A. (2014). Análisis de la eficacia de los saques de esquina en la copa del mundo de fútbol 2010. Un intento de identificación de variables explicativas. Revista de Psicología del Deporte, 1, 165172.

Bezerra, L. O., Sabino, F., Ferreira, V. H. G., y Vasconcellos, F. V. (2018). Comparative between football 7-a-side and football 11-a-side: Quantity and frequency of actions performed by goalkeepers. Revista Brasileira de Futsal e Futebol, 10, 300-307.

Boyko, R. H., Boyko, A. R., y Boyko, M. G. (2007). Referee bias contributes to home advantage in english premiership football. Journal of Sports Sciences, 25, 11851194. doi:10.1080/02640410601038576

Castellano, J., Perea-Rodríguez, A., y Hernández-Mendo, A. (2008). Análisis de la evolución del fútbol a lo largo de los mundiales. Psicothema, 4, 929-932.

Dicks, M., Uehara, L., y Lima, C. (2011). Deception, individual differences and penalty kicks: Implications for Goalkeeping in association football. International Journal of Sports Science \& Coaching, 6, 515-521. doi:10.1260/1747-9541.6.4.515

Dicks, M., Davids, K., y Button, C. (2010). Individual differences in the visual control of intercepting a penalty kick in association football. Human Movement Science, 29, 401-411. doi:10.1016/j.humov.2010.02.008

García-Ocaña, F. (1994). El portero de fútbol. España: Editorial Paidotribo.

García-Ocaña, F. (2008). El portero de fútbol. España: Editorial Paidotribo.

Hansen, C., Sanz-Lopez, F., Whiteley, R., Popovic, N., Ahmed, H. A., y Cardinale, M. (2017). Performance analysis of male handball goalkeepers at the World Handball championship 2015. Biology of Sport, 34, 393-400. doi:10.5114/biolsport.2017.6982

Ion, C., Serghei, S., y Constantin, C. (2018). Differentiated physical training within the framework of a yearly training cycle of young footballers specialized on the position of goalkeeper. Journal of Physical Education and Sport, 18, 270-275. doi:10.7752/jpes.2018.01036

Ibarrola, J. P. (2011). Manual técnico del portero de fútbol. España: Editorial Paidotribo.

Lago-Penas, C., y Lago-Ballesteros, J. (2011). Game location and team quality effects on performance profiles in professional soccer. Journal of Sports Science and Medicine, 10, 465-471.

Leite, W. (2017). Home advantage: comparison between the major European football leagues. Athens Journal of Sports, 4, 65-74. doi:10.30958/ajspo.4.1.4

Leo, F. M., Sánchez-Miguel, P. A., Sánchez-Oliva, D., Amado, D., y García-Calvo, T. (2014). Analysis of the group process and the performance in semiprofessional soccer. Revista Internacional de Medicina y Ciencias de la Actividad Física y el Deporte, 14, 153-168.

Lidor, R., Ziv, G., y Gershon, T. (2012). Psychological preparation of goalkeepers for the 11-m penalty kick in soccer: A review. Sport Psychologist, 26, 375-389. doi:10.1123/tsp.26.3.375

Martínez, V. (2001). Historia y estadística del fútbol español. España: Editorial Martínez Calatrava.

Mombaerts, E. (2000). Fútbol. Del análisis del juego a la formación del jugador. Barcelona: INDE.

Muñoz, S. P., Muñoz, R. D., Cayetano, A. R., García, S. L., y Muñoz, A. S. (2016). Estudio de las acciones técnicas del portero de fútbol profesional a lo largo de una temporada: Implicaciones para el entrenamiento. EmásF: Revista digital de educación física, 42, 22-37. 
Palau, J.M., López, M., y López, M. (2010). Relación entre eficacia, lateralidad, y zona de lanzamiento del penalti en función del nivel de competición en fútbol. Revista Internacional de Ciencias del Deporte, 19, 153-166. doi:10.5232/ricyde2010.01905

Pollard, R. (2008). Home advantage in football: A current review of an unsolved puzzle. The Open Sports Sciences Journal, 1, 12-14. doi:10.2174/1875399X00801010012

Rebelo-Goncalves, R., Figueiredo, A.J., Coelho-e-Silva, M.J., y Tessitore, A. (2016). Assessment of technical skills in young soccer goalkeepers: Reliability and validity of two goalkeeperspecific tests. Journal of sports science \& medicine, 15, 516-523.

Ruíz, C., Guerrero, A., y Garrido, A. (2007). Estudio comparativo de las habilidades técnico tácticas más frecuentes entre porteros de primera y segunda división española de fútbol durante la temporada 2004-2005. Revista Digital de Educación Física y Deportes, 11(105), 1-16.

Sainz de Baranda, P., Adán, L., García-Angulo, A., Gómez-López, M., Nikolic, B., y Ortega-Toro, E. (2019). Differences in the offensive and defensive actions of the goalkeepers at women's FIFA World Cup 2011. Frontiers in psychology, 10, 223. doi:10.3389/fpsyg.2019.00223

Sainz de Baranda, P., Llopis, L., y Ortega, E. (2005). Metodología global para el entrenamiento del portero de fútbol. España: Editorial Wanceulen.

Sainz de Baranda, P., y Ortega, E. (2002). Estudio comparativo de las acciones realizadas por los porteros de fútbol participantes en el Mundial de Francia '98 vs Eurocopa 2000. Efdeportes, 49.

Sainz de Baranda, P., Ortega, E., y Palao, J.M. (2008). Analysis of goalkeepers' defence in the World Cup in Korea and Japan in 2002. European Journal of Sport Science, 8, 127-134. doi:10.1080/17461390801919045

Sampaio, J., y Maçãs, V. (2012). Measuring tactical behaviour in football. International Journal of Sports Medicine, 33, 395-401. doi:10.1055/s-0031-1301320

Sarmento, H., Figueiredo, A., Lago-Peñas, C., Milanovic, Z., Barbosa, A., Tadeu, P., y Bradley, P. S. (2017). The influence of tactical and situational variables on offensive sequences during elite football matches. Journal of Strength and Conditioning Research, 32, 2331-2339. doi:10.1519/JSC.0000000000002147

Shamardin, V. N., y Khorkavyy, B. V (2015). Organizational structure of technical and tactical training of skilled goalkeepers in football. Pedagogics, psychology, medicalbiological problems of physical training and sports, 19, 75-79. doi:10.15561/18189172.2015.0213

Taylor, B. J., Mellalieu, D. S., James, N., y Barter, P. (2010). Situation variable effects and tactical performance in professional association football. International Journal of Performance Analysis in Sport, 10, 255-269. doi:10.1080/24748668.2010.11868520

Tenga, A., Holme, I., Ronglan, L. T., y Bahr, R. (2010). Effect of playing tactics on achieving score-box possessions in a random series of team possessions from Norwegian professional soccer matches. Journal of Sports Sciences, 28, 245-255. doi:10.1080/02640410903502766

Vales, A., Sambade, J., y Areces, A. (2002). Directrices metodológicas para el entrenamiento del portero de fútbol en alto rendimiento. Training fútbol: Revista técnica profesional, 77, 25-16.

White, A., Hills, S. P., Cooke, C. B., Batten, T., Kilduff, L. P., Cook, C. J., et al. (2018). Match-play and performance test responses of soccer goalkeepers: A review of current literature. Sports Medicine, 48, 2497-2516. doi:10.1007/s40279-018-09772

Número de citas totales / Total references: 39 (100\%).

Número de citas propias de la revista / Journal's own references: 2 (5\%). 
Rev.int.med.cienc.act.fís.deporte - vol. 20 - número 80 - ISSN: 1577-0354

Rev.int.med.cienc.act.fís.deporte - vol. X - número X - ISSN: 1577-0354 